\title{
Comparative Analysis and in vitro Experiments of Signatures and Prognostic Value of Immune Checkpoint Genes in Colorectal Cancer
}

\author{
Rui Ma ${ }^{\mathrm{I}-4}$ \\ Xiujuan $\mathrm{Qu}^{\mathrm{I}-4}$ \\ Xiaofang Che ${ }^{1-4}$ \\ Bowen Yang (iD) $1-4$ \\ $\mathrm{Ce} \mathrm{Li}^{1-4}$ \\ Kezuo Hou ${ }^{1-4}$ \\ Tianshu Guo ${ }^{1-4}$ \\ Jiawen Xiao $\mathbb{1 D}^{5}$ \\ Yunpeng Liu (iD) ${ }^{1-4}$ \\ 'Department of Medical Oncology, The \\ First Hospital of China Medical University, \\ Shenyang, I I000 I, People's Republic of \\ China; ${ }^{2}$ Key Laboratory of Anticancer \\ Drugs and Biotherapy of Liaoning Province, \\ The First Hospital of China Medical \\ University, China Liaoning Province Clinical \\ Research Center for Cancer, Shenyang, \\ I I000I, People's Republic of China; \\ ${ }^{3}$ Liaoning Province Clinical Research \\ Center for Cancer, Shenyang, I I000I, \\ People's Republic of China; ${ }^{4} \mathrm{Key}$ \\ Laboratory of Precision Diagnosis and \\ Treatment of Gastrointestinal Tumors, \\ Ministry of Education, Shenyang, II000I, \\ People's Republic of China; ${ }^{5}$ Department of \\ Medical Oncology, Shenyang Fifth People \\ Hospital, Shenyang, People's Republic of \\ China
}

Correspondence: Yunpeng Liu; Xiujuan Qu

Department of Medical Oncology, The First Hospital of China Medical University, No. 155, North Nanjing Street, Heping District, Shenyang, I 1000I, People's

Republic of China

Tel +86-24-832823/2

Fax +86-24-83282543

Email ypliu@cmu.edu.cn;

xiujuanqu@yahoo.com
Purpose: Immune checkpoints, as pivotal regulators of immune escape in cancer, can motivate the emergence of immune checkpoint inhibitors (ICIs). The aim of this study is to identify the expression of the immune checkpoint genes (ICGs) in colorectal cancer (CRC) and to relate their individual as well as combined expression to prognosis and therapeutic effectiveness in CRC.

Methods: RNA expression of 47 ICGs and clinical information of CRC patients were collected from two public databases to elucidate the expression levels and prognostic values of these ICGs in CRC. Then, the Shapiro-Wilk normality test was used to determine the normality of variables. Overall survival (OS) rates of each subset were found by KaplanMeier method, and the statistical significance was determined by the Log rank test $(p<0.05)$. Results: The expression of 13 and 9 ICGs was significantly associated with CRC prognosis in The Cancer Genome Atlas (TCGA) and Gene Expression Omnibus (GEO) cohorts. A series of ICGs was found to be significantly associated with TMB, neoantigens and MMR in CRC indicating that the combination of immunotherapy treatment biomarkers and ICGs may achieve accurate prognostic stratification of $\mathrm{CRC}$, and potentially identify $\mathrm{CRC}$ cases that might respond to checkpoint inhibitors (CPIs). The subsets of high or low PD1/PD-L1/IDO1 expression stratified by CD48 were accurately associated with prognosis in CRC. In addition, in vitro experiments confirmed that VTCN1(B7-H4)-KD increases antiPD-L1-mediated NK cell cytotoxicity on CRC tumor cells.

Conclusion: Although the expression of a single immune-checkpoint molecule does not predict the efficacy of immunotherapy in CRC, our findings infer that subsets defined by ICGs are associated with prognosis and imply the possibility that VTCN1 and CD48 serve as new immunotherapeutic targets.

Keywords: immune checkpoint genes, colorectal cancer, prognosis, immunotherapy

\section{Introduction}

Colorectal cancer (CRC) is the third most commonly diagnosed malignancy and cause of cancer-related deaths. ${ }^{1}$ Despite significant improvements in CRC detection and treatment, the prognostic outcome of patients with metastatic CRC (mCRC) remains poor. ${ }^{2}$ Therefore, it is necessary to identify novel prognosis biomarkers and carry out more effective treatments for improving the outcome of patients with CRC.

In recent years, it has been found that the immune system plays a crucial role during cancer initiation, progression and metastasis. ${ }^{3,4}$ The activity of the immune 
system is mainly regulated by different types of immune cells. The activity and response of immune cells are modulated by a series of cell surface molecules, named immune checkpoints including stimulatory and inhibitory checkpoint molecules. ${ }^{5}$ Based on the most broadly studied inhibitory checkpoints, the development of ICIs, such as antiCTLA-4 monoclonal antibodies (mAbs), anti-PD-1/PD-L1 mAbs have revolutionized several cancer treatment paradigms. ${ }^{1}$ In addition, agonist agents targeting co-stimulatory receptors have been evaluated in various preclinical settings and are being studied in multiple clinical trials of several types of cancers. ${ }^{6}$

Despite the pronounced survival benefits conferred by ICIs, not all CRC patients respond to immunotherapy due to the complexity of the immune microenvironment and the diversity of immune checkpoint molecules. Only different Mismatch Repair (dMMR) or microsatellite instability-high (MSI-H) mCRC patients responded to targeted immune checkpoint inhibition therapy. ${ }^{7}$ Since only approximately $4 \%$ of advanced CRC patients have MSI or dMMR, ${ }^{8-11}$ majority of mCRC patients with pMMR or MSS tumours do not benefit from immunotherapy. Besides, as some patients with dMMR did not benefit from treatment, MMR status might not be the only positive predictor for anti-PD-1 therapy for CRC, some other predictor should be explored for use in combination with MMR status for better predicting the therapeutic effect. $^{12-15}$ Notably, the association has been reported between immune checkpoint molecules and prognosis. ${ }^{6,16-19}$ However, the prognostic significance of these immune checkpoint molecules remains controversial in CRC. Therefore, better understanding of the characteristics and prognostic significance of immune checkpoint molecules is critical to improve the efficacy of immunotherapy in cancer. Additionally, evidence has shown that up-regulation of other inhibitory molecules, such as LAG-3, VISTA, and TIM-3 may generate tumour resistance to specific ICIs. ${ }^{20-22}$ Also, the blockade of a single immune checkpoint targets may lead to compensatory upregulation of other checkpoint receptors in the tumour microenvironment (TME) ${ }^{23}$ Realizing the relationship of different immune checkpoints will benefit the design of effective combination therapies and overcome potential resistance; however, existing studies have focused on one or several immune checkpoint molecules without combined evaluation of them in CRC.

In this study, we aimed to analyze the expression of 47 ICGs of CRC and their relationship with prognosis, using the TCGA and GEO databases. Furthermore, we examined the association of ICGs with other immunotherapy biomarkers and signature genes related to immune activation in CRC. The findings have implications for our understanding of the pre-treatment predictive biomarkers for immunotherapy of CRC, and for development of new immunotherapy approaches for CRC.

\section{Materials and Methods}

\section{Data Download}

A total of 47 ICGs of $\mathrm{CRC}^{24}$ were downloaded (Supplementary Table 1). TCGA GDC API was used to download the latest clinical follow-up information at 2019.6.14 containing 454 RNA-seq data samples (Supplementary Table 2). Download GSE39582 chip expression data in TXT from NCBI. GSE39582 contains 585 samples with clinical characteristics (Supplementary Table 3).

\section{Data Preprocessing}

Data Retrieval and Preprocessing in TCGA Database

The 454 samples of RNA-seq data were dealt as the following steps:

1. Use GDC API to download the RNA-seq data set of CRC from TCGA.

2. Select the original samples.

3. Remove the samples with no follow-up data and the samples with a follow-up time less than 30 days.

4. Remove samples with no clinical information or PFS $<30$ days.

5. Remove the normal tissue sample data.

6. Remove the gene with 0 FPKM from more than half of the samples.

The sample number of TCGA data set was 428 after preprocessing.

\section{Data Retrieval and Preprocessing in GEO Database}

The 585 samples of RNA-seq data were dealt as the following steps:

1. Remove the normal tissue sample data and primary tumor data were retained.

2. Convert OS data to days from years or months.

3. Bioconductor package were used to annotate the microchip probes of the human gene SYMBOL.

4. The immune-related genes expression profiles were obtained. 
The sample number of GSE39582 data set was 556 after preprocessing.

Detailed clinical characteristics of all patients from the two data sets are listed in Table 1.

\section{Correlation Between ICGs and Patient Prognosis}

The ICGs with expression in TCGA dataset were identified. The cases were divided into three (high, middle, low) groups based on levels of gene expression. The correlation between ICGs and CRC prognosis was performed by univariate Cox analysis (log rank $p<0.05$ ). Then GEO database was used to conduct the expression and prognostic patterns of the ICGs in CRC.

\section{Correlation Between ICGs and TMB and Neoantigens}

TMB represents both the stability level of the tumor genome and heterogeneity of the tumor micro-environment. TMB of a tumor sample is calculated by the number of non-synonymous somatic mutations (single nucleotide variants and small insertions/deletions) per mega-base in coding regions. We used the WES data of TCGA and used the same method as $\mathrm{Xu}$ et $\mathrm{al}^{25}$ to assess the total length of all mutations divided by exons in each sample. Spearman method was used to evaluate the correlation among neoantigens, TMB and ICGs based on the somatic mutation data of TCGA.

\section{Correlation Between Subtypes Defined by ICGs and Prognosis}

We first integrated the expressions of IDO1, CD274, CTLA4 and $\mathrm{CD} 48$, respectively. COAD samples were then divided into multiple subtypes based on these gene expression values. Finally, the survival prognosis of subtypes of different gene expression combinations was analyzed. Kaplan-Meier survival curve was drawn by Survfit function in survival software package, and the test method was Log rank $p$.

\section{Cell Culture}

Human CRC SW-620, HCT-116 and Caco-2 cell lines were purchased from the Type Culture Collection of the Chinese Academy of Sciences (Shanghai, China) and cultured in RPMI 1640 medium. DiFi cell line was purchased from Shanghai Bai Li Biological Technology Co., Ltd (Shanghai, China) and cultured in MEM medium. All medium contained $10 \%$ heat-inactivated fetal bovine serum (FBS), penicillin $(100 \mathrm{U} / \mathrm{mL})$, and streptomycin $(100 \mu \mathrm{g} / \mathrm{mL})$ in an atmosphere of $95 \%$ air and $5 \% \mathrm{CO}_{2}$ at $37^{\circ} \mathrm{C}$. NK-92 was obtained from Nanjing Forxay Biotechnology Co. LTD (Nanjing, China) and cultured in MEM supplemented with $12.5 \%$ horse serum (GIBCO Invitrogen), 12.5\% fetal bovine serum (BI), $100 \mathrm{Uml}^{-1}$ rhIL-2, $0.1 \mathrm{mM}$ 2-mercaptoethanol, $0.2 \mathrm{mM}$ inositol and $0.02 \mathrm{mM}$ folic acid. Cells were routinely passed every $2-3$ days and all cells maintained in culture for a maximum 8 weeks.

\section{Reagents and Antibodies}

SHR-1316 (anti-PD-L1) was purchased from Hengrui Medicine (Lianyungang, China). Antibodies of B7-H4 (14572S) and GAPDH (5174S) were obtained from Cell Signaling Technology (Danvers, MA, USA).

\section{Flow Cytometry Analysis}

For testing the B7-H4 surface expression, human CRC cells were harvested and washed with PBS then incubated with PE Mouse-Anti-Human-B7-H4 (BD, Cat \#562,507) or isotype control antibody for $30 \mathrm{~min}$ at $4{ }^{\circ} \mathrm{C}$ avoiding light. Cells were then washed and analyzed on flow cytometry (BD Biosciences).

\section{Co-Cultures with NK-92 Cells and NK Cell Cytotoxicity Activity Test}

NK cell lysis of target CRC cells was determined by ELISA and flow cytometry after $24 \mathrm{~h}$ of co-incubation with or without SHR-1316. Then to detect NK activation, the expression of soluble IFN- $\gamma$ in culture was detected by enzyme-linked immunosorbent assay (ELISA). To study apoptosis, cells were stained with AnnexinV-FITC/7AAD (BD Biosciences, USA). The CD56-APC antibody (BD, Cat $\# 555,518$ ) was used to identify NK cells and the level of apoptosis was calculated for the CD56- cells. Next, to measure the level of NK cell degranulation, target cells and NK cells at 1:1 E/T ratio incubated with CD107a-PE (BD, Cat $\# 555,801$ ) for $4 \mathrm{~h}$. The percentage of CD107a positive cells was calculated for the $\mathrm{CD} 56+$ cell fraction.

\section{Small Interfering RNA Transfection}

The siRNA sequence was designed by View Solid Biotechnology Co., LTD (Beijing, China). The B7-H4 target sequence was 5'-GGUGUUUUAGGCUUGGUCC3'. The siRNAs were transfected with Lipofectamine 2000 (Invitrogen, USA) per the manufacturer's instructions. 
Table I Clinical Characteristics of Patients with CRC from the Two Data Sets After Pre-Processing

\begin{tabular}{|c|c|c|c|}
\hline TCGA & & GEO & \\
\hline SEX & & SEX & \\
\hline MALE & 231 & MALE & 307 \\
\hline FEMALE & 197 & FEMALE & 249 \\
\hline PFS & & OS & \\
\hline $30 \sim 925$ & 298 & $30 \sim 1230$ & 206 \\
\hline $925 \sim 1820$ & 96 & $1230 \sim 2430$ & 212 \\
\hline $1820 \sim 27 \mid 5$ & 21 & $2430 \sim 3630$ & 97 \\
\hline $2715 \sim 3610$ & 3 & $3630 \sim 4830$ & 31 \\
\hline $3610 \sim 4502$ & 10 & $4830 \sim 6030$ & 9 \\
\hline Event & & Event & \\
\hline Dead & 115 & 0 & 369 \\
\hline Alive & 313 & I & 187 \\
\hline Stage $T$ & & Stage $T$ & \\
\hline TI & 10 & TO & 1 \\
\hline $\mathrm{T} 2$ & 75 & TI & 11 \\
\hline T3 & 295 & T2 & 44 \\
\hline \multirow[t]{2}{*}{$\mathrm{T} 4$} & 47 & T3 & 360 \\
\hline & & $\mathrm{T} 4$ & 117 \\
\hline Stage N & & Stage $N$ & \\
\hline No & 252 & No & 295 \\
\hline $\mathrm{NI}$ & 101 & NI & 131 \\
\hline \multirow[t]{2}{*}{ N2 } & 75 & N2 & 98 \\
\hline & & N3 & 6 \\
\hline Stage M & & Stage $M$ & \\
\hline Mo & 318 & Mo & 474 \\
\hline MI & 59 & MI & 60 \\
\hline$M X$ & 51 & $M X$ & 22 \\
\hline Stage & & TNM stage & \\
\hline Stage I & 72 & 1 & 32 \\
\hline Stage II & 164 & 2 & 258 \\
\hline Stage III & 122 & 3 & 203 \\
\hline Stage IV & 59 & 4 & 59 \\
\hline \multicolumn{4}{|l|}{ Weight } \\
\hline $34 \sim 63$ & 48 & & \\
\hline $63 \sim 92$ & $13 \mid$ & & \\
\hline $92 \sim 121$ & 53 & & \\
\hline $121 \sim 150$ & 7 & & \\
\hline $150 \sim 175$ & 3 & & \\
\hline Not Available & 186 & & \\
\hline \multicolumn{4}{|l|}{ BMI } \\
\hline $14.7 \sim 66.7$ & 225 & & \\
\hline $66.7 \sim 118.7$ & 0 & & \\
\hline I I $8.7 \sim 170.7$ & 0 & & \\
\hline $170.7 \sim 222.7$ & 0 & & \\
\hline $222.7 \sim 271.8$ & I & & \\
\hline
\end{tabular}

(Continued)
Table I (Continued).

\begin{tabular}{|l|c|l|c|}
\hline TCGA & & GEO & \\
\hline New Event & & & \\
I & 87 & & \\
0 & 341 & & \\
\hline Site of resection & & Tumor location & \\
Ascending colon & 76 & Distal & 338 \\
Cecum & 87 & Proximal & 217 \\
Colon, NOS & 94 & & \\
Descending colon & 16 & & \\
Hepatic flexure of colon & 15 & & \\
Rectosigmoid junction & 7 & & \\
Sigmoid colon & 106 & & \\
Splenic flexure of colon & 5 & & \\
Transverse colon & 19 & & \\
\hline Chemotherapy & & Chemotherapy & \\
5FU & 31 & 5FU & \\
FOLFIRI & 3 & FOLFIRI & \\
FOLFOX & 5 & FOLFOX & 23 \\
FUFOL & 0 & FUFOL & 53 \\
Other & 115 & Other & \\
NA & 276 & NA & \\
\hline MSI Score & 339 & & \\
$0.25-0.5$ & 43 & & \\
$0.5-I .0$ & 18 & & \\
I.0-I.5 & & & \\
& & \\
\hline
\end{tabular}

\section{Western Blot Analysis}

Western blot was carried out according to the method in the previous study. ${ }^{26}$ The antibodies used in this experiment have been described previously.

\section{Real-Time Quantitative PCR Analysis}

Total RNA was isolated from transfected CRC cells using Trizol reagent (Takara Bio, Japan) and following we reversed RNA transcriptions into stable cDNA using PrimeScript RT Reagent Kit with gDNA Eraser (Takara Bio). The cDNA was amplified using SYBR Premix Ex Taq II Kit (Takara Bio). Real-time reactions and PCR steps were performed according to the manufacturer's instructions. The primer sequences were as follows: B7-H4: 5' CACCAGGATAACATCTCTCAGTGAA-3' (forward), 5'TGGCTTGCAGGGTAGAATGA -3' (reverse); 18S: 5'CCCGGGGAGGTAGTGACGAAAAAT-3' (forward), 5'CGCCCGCCCGCTCCCAAGAT-3' (reverse). 


\section{Cell Viability Assay}

The viability assays in HCT-116 cells co-incubated with NK were measured using a MTT assay. Briefly, after infected with siRNA, co-incubated cells (10:1 E/T ratio) were added to 96 well culture plates and incubated overnight. Then, the cells were pretreated with no treatment or SHR-1316 for $24 \mathrm{~h} .20 \mu \mathrm{L}$ MTT solution was added to each well and cultured for $4 \mathrm{~h}$ followed by $200 \mu \mathrm{L}$ DMSO (Sigma) added. At last, the absorbance at $570 \mathrm{~nm}$ was determined by microplate reader (Bio-Rad, USA).

\section{Ethical Statement}

The study was approved by the Ethics Committee of the Affiliated Hospital of China Medical University (Shenyang, China) and was conducted according to the Declaration of Helsinki.

\section{Statistical Analysis}

All the normality of variables was conducted by ShapiroWilk normality test. Nonpaired Student's $t$-test was used to determine the statistical significance of the normal distribution variables. Moreover, the Mann-Whitney $U$-test was used to evaluate the non-normal distribution variables. When comparing more than two groups, we used nonparametric and parametric methods by Kruskal-Wallis test and one-way ANOVA, respectively. The association between ICGs and other biomarkers in the entire cohort was evaluated using the Spearman rank correlation analysis. The Fisher's exact test was applied to determine the contingency table. The P-value was adjusted using the Benjamini-Hochberg procedure. Survival analysis was performed using Kaplan-Meier, and the survival differences were calculated using the Log rank test at the level of $p<0.05$. All analyses were conducted using R software (version 3.4.3).

\section{Results}

\section{Construction of ICGs and Their Prognostic Value for CRC in the TCGA Cohort} Expression of ICGs in CRC

Firstly, a total of 43 ICGs of CRC with expression profile were extracted from the data set pre-processed by TCGA database. The expression level of 43 ICGs is shown in Figure 1A. These included 12 stimulatory molecules (TNFRSF14, TNFSF14, ICOS, ICOSLG, CD70, CD27, CD48, TNFRSF9, TNFRSF4, THFRSF25, THFSF9 and
CD40) and 31 inhibitory checkpoint molecules. Heatmap showed distinct gene expression profiles of ICGs belong to high expression group (red), medium expression group (green) and a low expression group (blue). The high expression group was represented by CD44, LGALS9, TNFRSF14, the expression level was usually higher in all samples. The Intermediate expression group, expressed by IDO1, HHLA2 and TNFSF9, varied widely from sample to sample. The low expression group was represented by ADORA2A, IDO2, etc., and the expression level was low in most samples. The results suggested that most ICGs showed medium and low expression in CRC samples.

\section{Identification of CRC Specific ICGs for Prognosis Prediction}

Overall, we found that the expression of ICGs was significantly associated with CRC prognosis. According to the univariate Cox regression analysis between 43 expressed ICGs and prognosis, a total of five were found to be significantly correlated with prognosis ( $\log \operatorname{rank} p<$ 0.05 ) and eight to be marginal significantly correlated with prognosis (Figure 1B, log rank $p<0.1$ ). Correlations around all ICGs were also assessed. Results showed the level of expression of ICGs was mainly positively correlated and had significant aggregation effect (Figure 1C), implying the co-expression relationship between ICGs. Therefore, the results confirmed the synergistic prognostic values of the ICGs in CRC.

\section{Construction of ICGs and Its Prognostic Value for CRC in the GEO Cohort}

In order to avoid the bias from only examining the TCGA cohort, we used GSE39582 as a validation dataset. Fortyseven ICGs were taken with a total of 44 expressions in the pre-processed GSE39582 set, and the gene expression patterns of these 44 ICGs were also grouped into three types: high/middle/low expression group, respectively (Figure 2A). Furthermore, the expression of genes in different groups of GSE39582 set were consistent with that in the TCGA. Such as CD44, LGALS9 and TNFRSF14 exhibited high expression levels in both data sets. TNFSF15, TNFSF9, CD86, CD200, TNFRSF18 and HAVCR2 showed medium expression levels in both data sets. Meanwhile, VTCN1, CD28, IDO2, TIGIT, TNFRSF9, and TNFSF4 had low expression levels in both data sets.

In univariate Cox regression analysis of 44 ICGs and overall survival (OS), we found that there are nine ICGs 


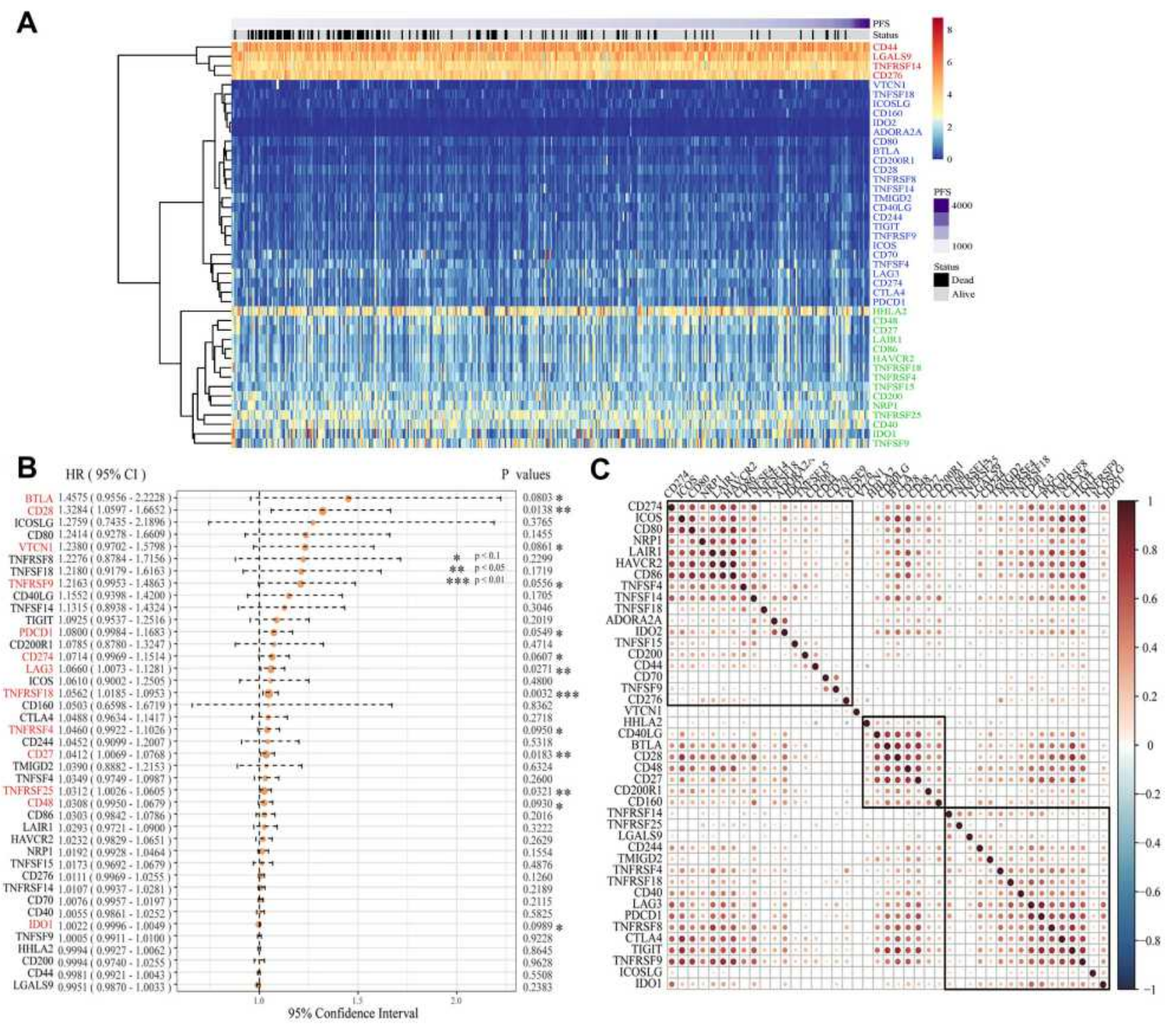

Figure I Construction of ICGs and its prognostic value for colorectal cancer in the TCGA cohort. (A) A heatmap delineated the expression of ICGs in CRC on TCGA dataset. Red: high expression groups, green: medium expression groups; Blue: low expression groups; (B) The expression of I3 ICGs associated with CRC prognosis; (C) Correlation analysis of ICGs expression level. Explanation: We only offer the gene pairs with significant correlation test, meanwhile the blank indicated that the correlation test was not significant (Data are plotted as mean $\pm \mathrm{SD}$. $* \mathrm{P}<0 . \mathrm{I},{ }^{*} * \mathrm{P}<0.05, * * * \mathrm{P}<0.0 \mathrm{I}$ ).

associated with OS (Figure 2B). There is a possibility that VTCN may be one of the potential markers for poor prognosis, because the VTCN1 gene showed a negative correlation with prognosis in both TCGA and GSE39582 data set (HR $>1, \log$ rank $p<0.1$ ). Simultaneously, TNFRSF25, CD48 and IDO1 genes in the TCGA and GSE39582 data sets all had significant or marginal relationship with the prognosis (log rank $p<0.1)$. In the GSE39582 dataset, the expression levels of ICGs were also mainly positively correlated and exhibited an aggregation effect (Figure 2C), which is consistent with the result of TCGA.
Relationship Between ICGs and Other Immunotherapy Treatment Biomarkers in CRC

\section{Relationship Between ICGs and TMB in CRC}

Tumour mutational burden (TMB) is one of the biomarkers for predicting the efficacy of immunotherapy, ${ }^{27}$ however, it is still unclear as to whether a high TMB could be confirmed as a positive prognostic marker in mCRC. Spearman's method was performed to access the correlation between TMB and ICGs (the distribution of TMB did not meet the normal distribution, Shapiro test 


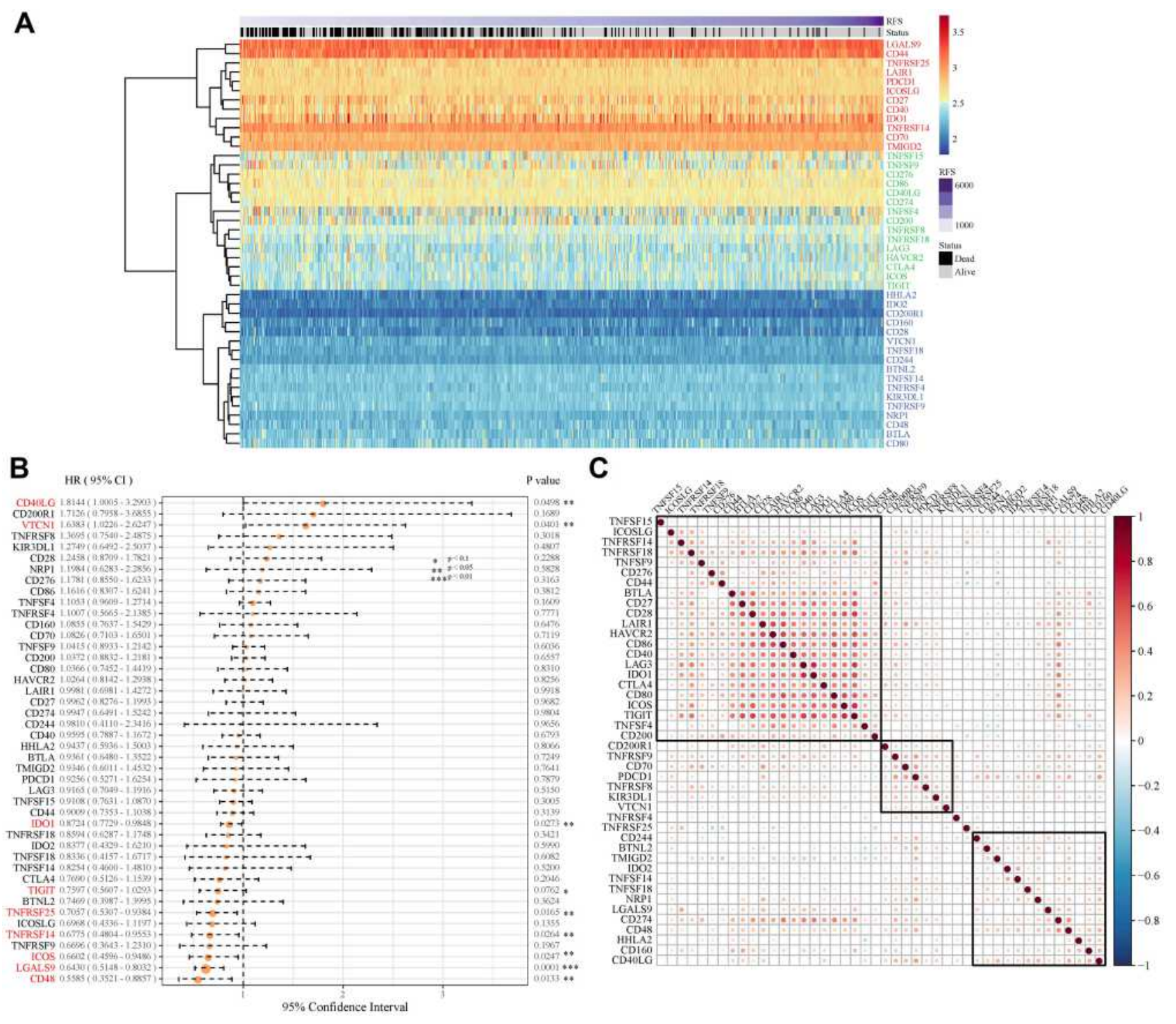

Figure 2 Construction of ICGs and its prognostic value for colorectal cancer in the GEO cohort. (A) ICGs express heatmap in GSE39582 dataset. Red: high expression group, green: medium expression group; Blue: low expression group. (B) The expression of 9 ICGs associated with CRC prognosis; (C) Correlation of ICGs expression level. Explanation: only the gene pairs with significant correlation test were shown, and the blank indicated that the correlation test was not significant (Data are plotted as mean \pm SD. $* \mathrm{P}<0.1, * * \mathrm{P}<0.05$, $* * * \mathrm{P}<0.01)$.

$\mathrm{p}<1 \times 10^{-5}$ ). The expression data pertaining to TMB and ICG are shown in Supplementary Table 4. We observed a significantly positive correlation between TMB and the expression of VTCN1, CD274, IDO1, LAG3, PDCD1 and TNFRSF9 (Figure 3A-F, $R^{2}>0$ and FDR $<0.05)$. Considering that TMB is a biomarker of positive prognosis, subgroup analysis can be combined with ICGs to predict prognosis more accurately in CRC.

Relationship Between ICGs and Neoantigens in CRC Neoplastic somatic mutations, when present in the protein-coding genes, produce the wrong proteins (neoantigens) and accumulate by cancer cells during the tumorigenesis process. Neoantigens are now supposed to be able to participate in early tumor recognition and destruction by antigen-specific $\mathrm{T}$ cells in the context of immunotherapy treatment. The relationship between neoantigens and ICGs expression from TCGA was shown in Supplementary Table 5. We found that CD48, CD274, IDO1, LAG3, PDCD1 and TNFRSF9 also had significantly positive correlation with neoantigens (Figure 3G-L, $R^{2}>0.2$ and FDR $<0.001$ ), which was consistent with the significantly positive correlation between TMB and CD274, IDO1, LAG3, PDCD1 and TNFRSF9 as mentioned above. 

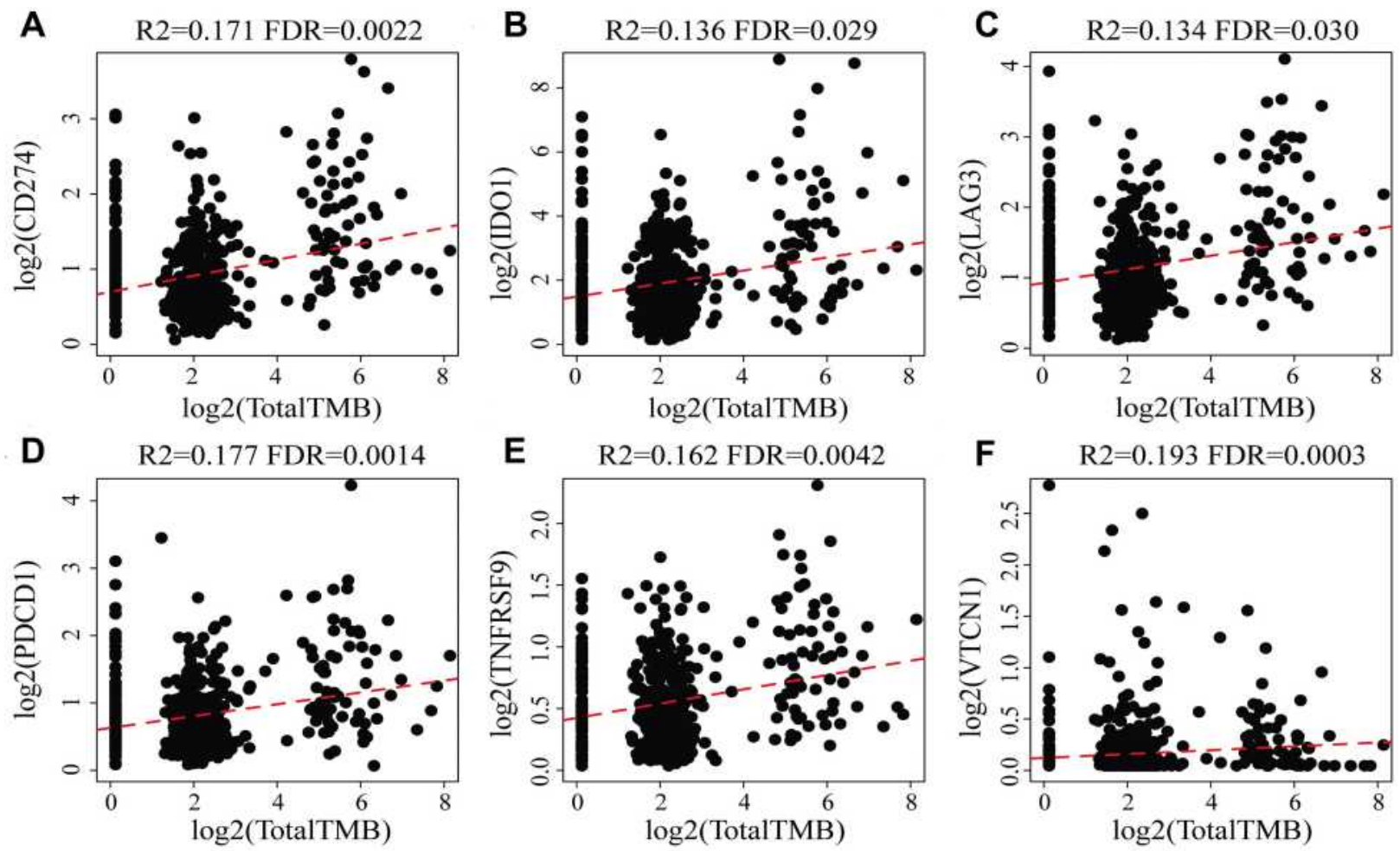

G $\mathrm{R} 2=0.226 \mathrm{FDR}=0.00016$

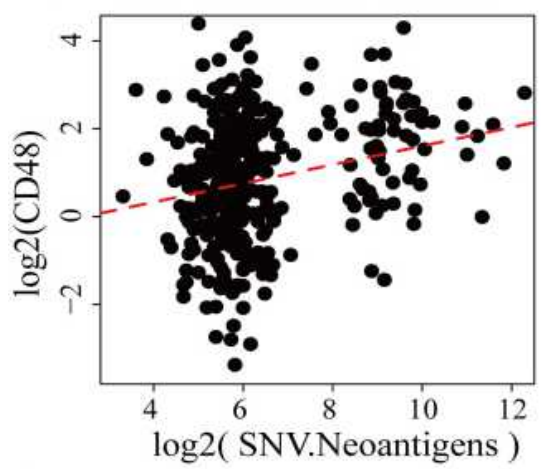

H R2 $=0.380$ FDR $=3.92 \mathrm{e}-12$
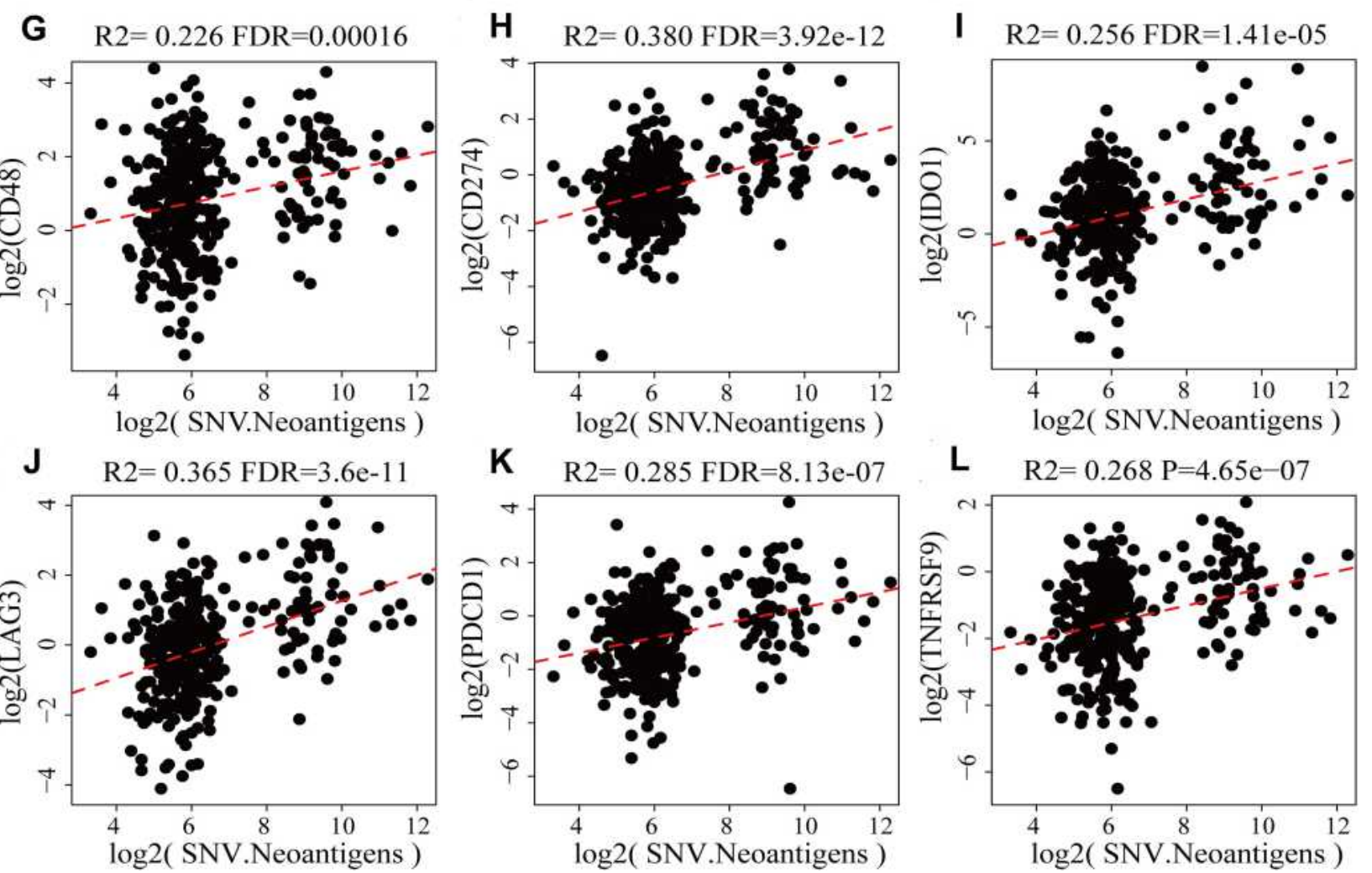

K R2 $=0.285$ FDR $=8.13 \mathrm{e}-07$
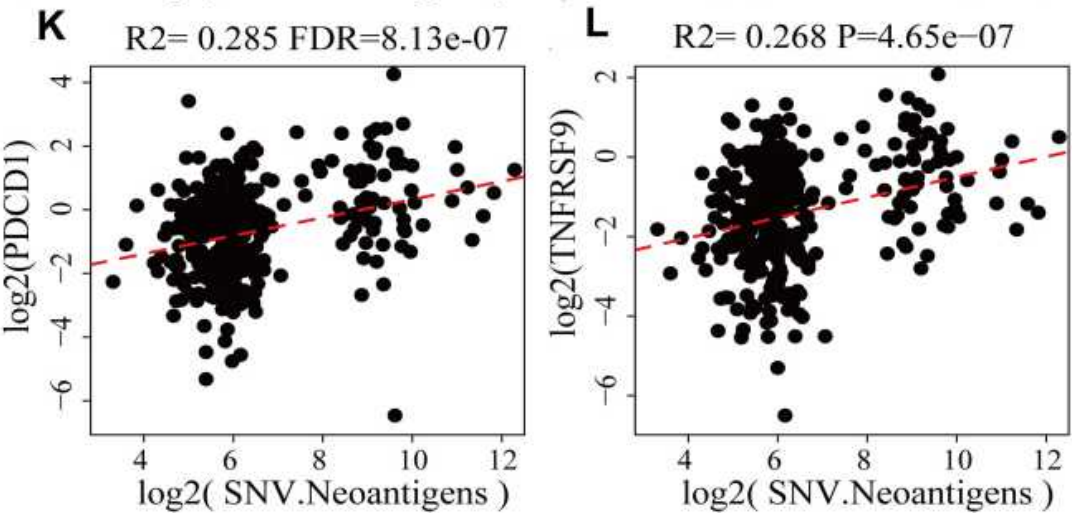

Figure 3 Scatter diagram between the expression levels of ICGs and TMB and neoantigens. $R^{2}$ is the correlation coefficient, and FDR is the false-positive detection rate. The $\mathrm{x}$-coordinate represents the expression for TMB/SNV. The ordinate represents gene expression. (A-F) are the scatter diagram between CD274, IDOI, LAG3, PDCDI, TNFRSF9, VTCNI and TMB respectively; (G-L) are the scatter diagram between CD48, CD274, IDOI, LAG3, PDCDI, TNFRSF9 and SNV respectively. 


\section{Relationship Between ICGs and MMRs in CRC}

MMRs is an intracellular mismatch repair mechanism in which the loss of key genetic function results in DNA replication errors that cannot be repaired, leading to the generation of higher somatic mutations. In the present study, COAD somatic mutation data from TCGA was used to assess the association between MMRs genes (MLH1, MSH2, MSH6, PMS2, EPCAM) mutation and the expression of ICG. Among them, 43 samples had different numbers of MMRs mutants, the mutation information of specific MMRs is shown in Supplementary Table 6. We found that the expression level of MMRs was positively correlated with ICGs. In addition, TNFRSF9 was significantly correlated with MMRs (Figure 4, R > 0.25), which was markedly correlated with prognosis, TMB and neoantigens.

\section{Relationship Between ICGs and Immune Cell Subset in CRC}

CD8 $+\mathrm{T}$ cells in the TME can produce interferon-gamma (IFN $\gamma$ ), leading to up-regulation of adaptive immune-resistance pathways, including the PD-1/PD-L1 axis and IDO1, etc. ${ }^{24}$ Therefore, we explored whether expression of immune subset genes, such as CD8A, GZMB, CD68 and NOS2 in the TME was associated with expression of ICGs (Supplementary Table 7).

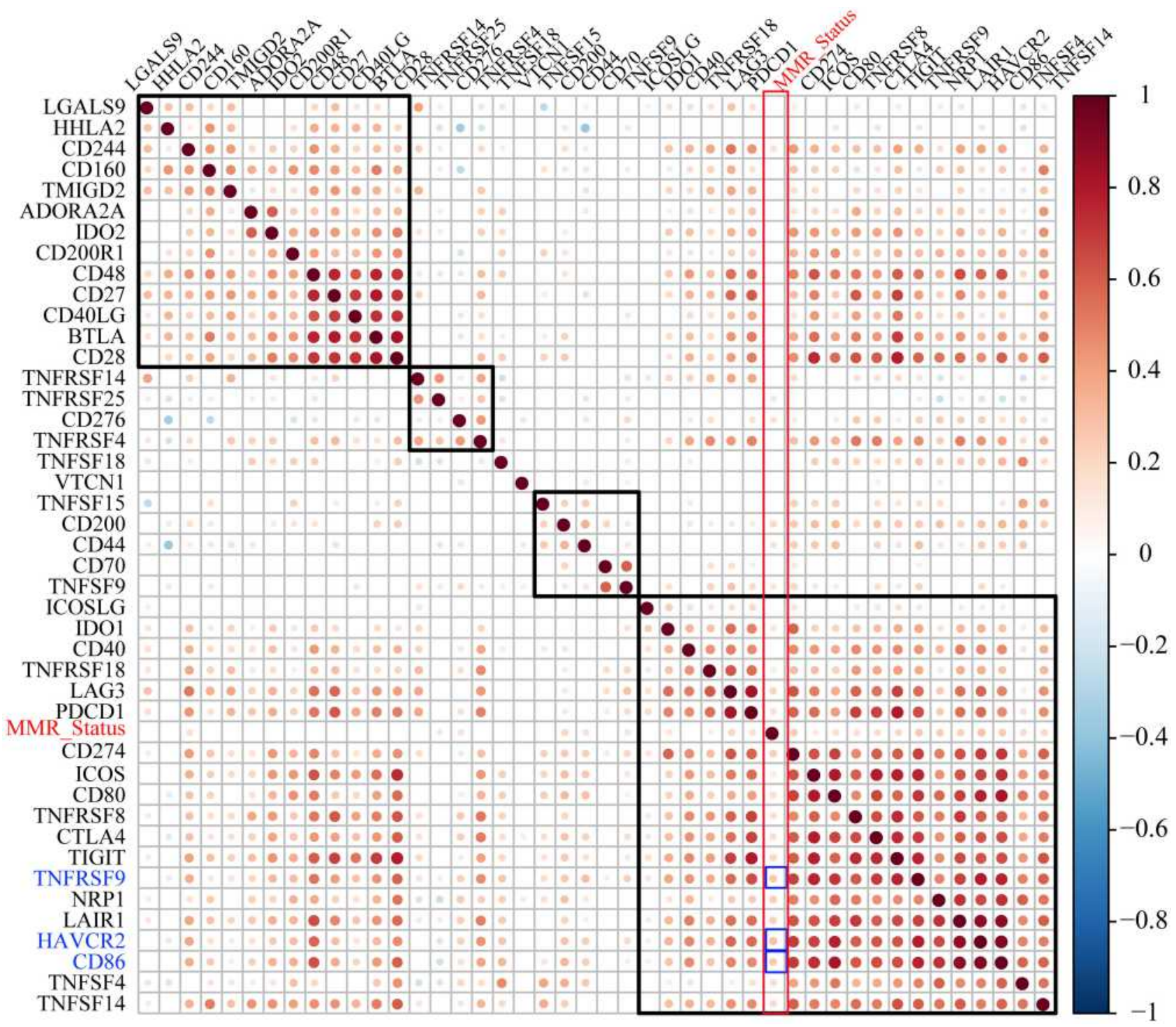

Figure 4 The correlation between the expression levels of ICGs and MMR gene mutation, the larger the point, the stronger the correlation. The redder the color in the figure, the stronger the positive correlation; The bluer the color, the stronger the negative correlation; The whiter the color, the weaker the correlation. 
The results showed that CD8A gene was highly correlated with the expression of ICGs (Figure 5A), and most of the genes were positively correlated with one another. The significance test of correlation coefficients showed that most of the correlations between these genes were extremely significant ( $p<R^{2}$, Figure 5B).

Using GSE39582 data as a validation dataset, we also examined the expression relationship between immune subsets and ICGs (Supplementary Table 8). As expected, a positive relationship between them was observed. CD8A was significantly positively correlated with TIGIT, LAG3, IDO1, and CD27 (Supplementary Figure 1), which was consistent with the data set of TCGA.
Notably, the prognostic value of ICGs combined with other immunotherapy treatment biomarkers in CRC is context-dependent and requires further confirmation.

\section{Association Between ICGs and Clinical Features in CRC}

The better to understand the prognostic value and underlying mechanisms of these ICGs, we further investigated the relationships between ICGs expression and clinicpathological factors in CRC. Combined 13 ICGs that were significantly associated with prognosis with clinical information provided by TCGA, we analyzed the expression patterns of these 13 genes and clinical characteristics
A

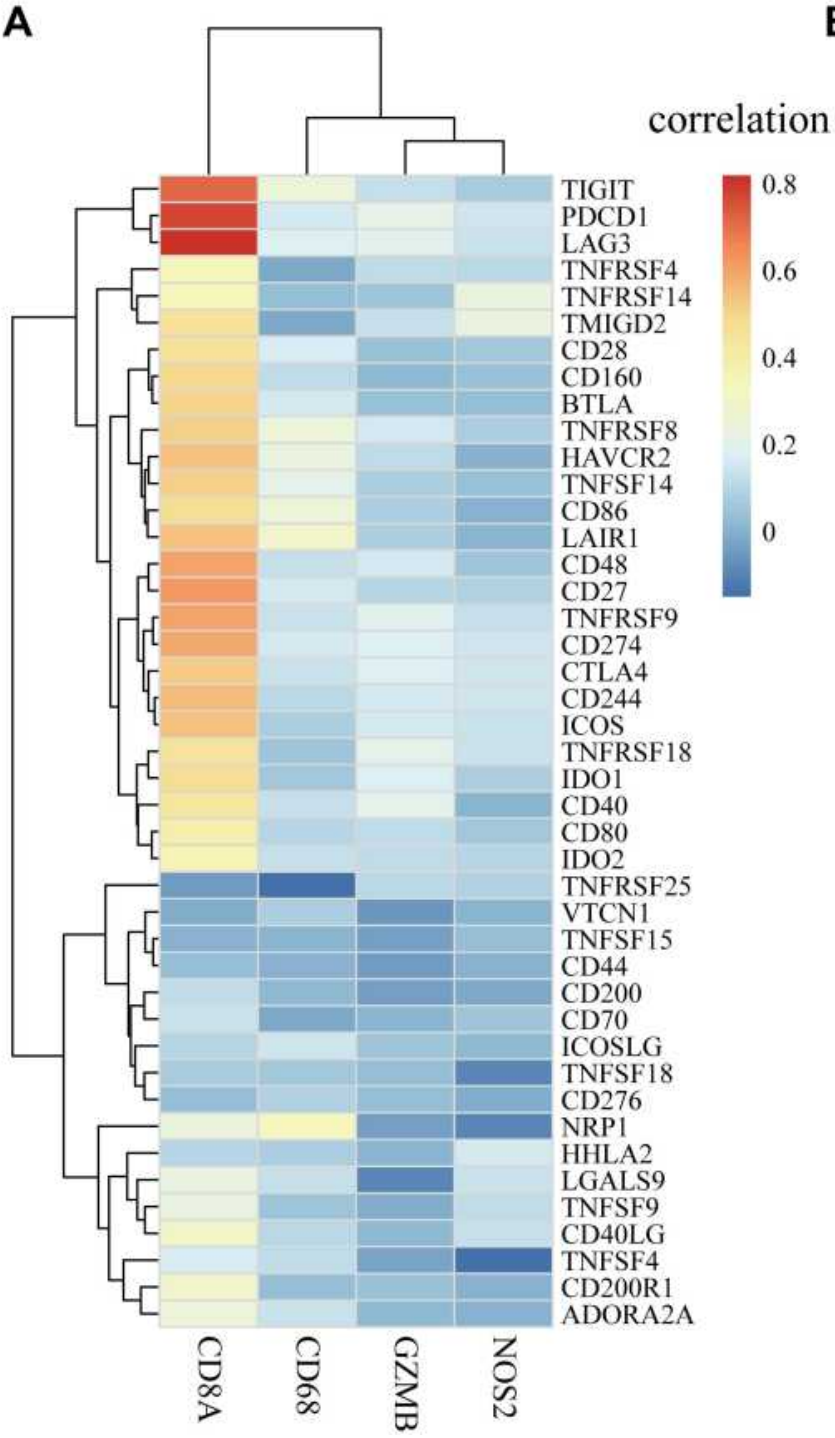

B

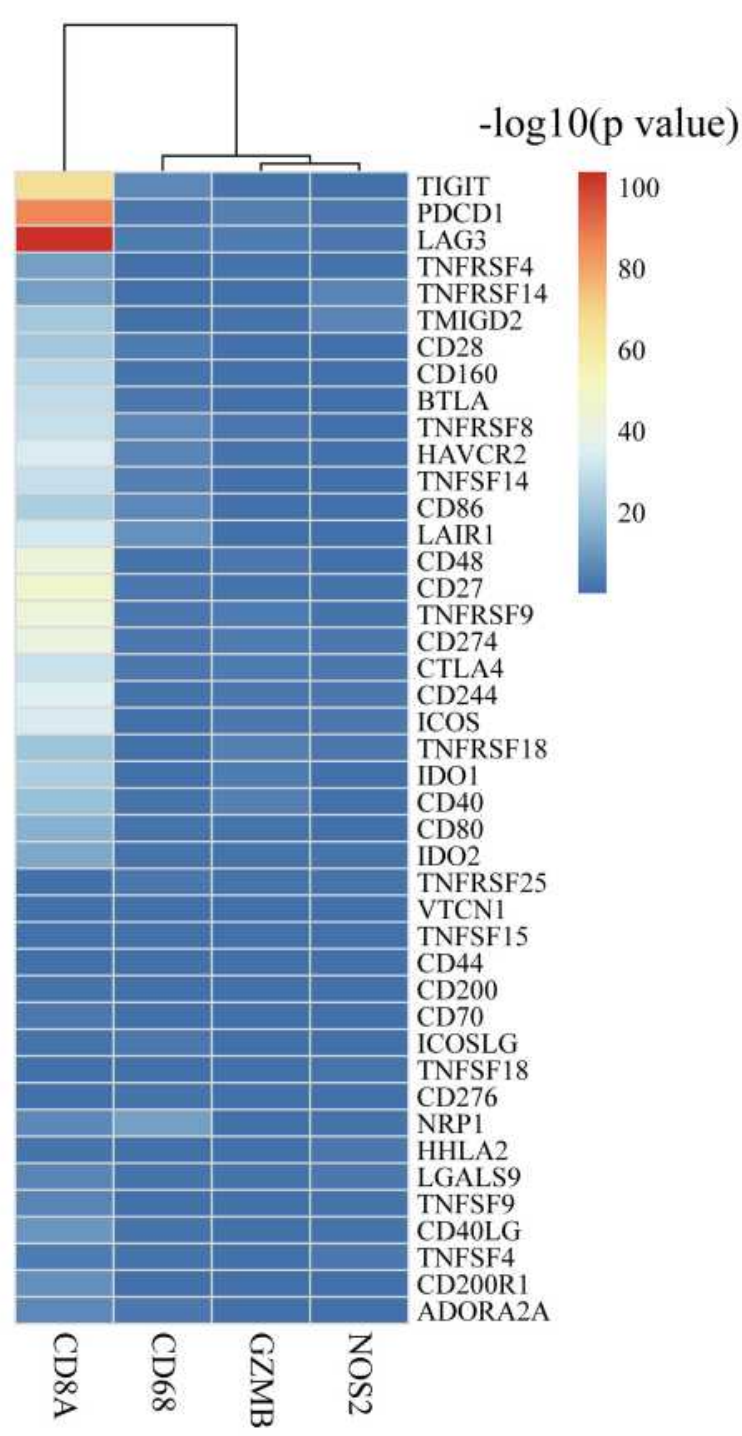

Figure 5 The correlation between ICGs and immune cell subsets in TCGA cohort. (A) Heat map of correlation coefficient between ICGs and immune cell subsets including CD8A, GZMB, CD68 and NOS2; (B) P-value test of correlation coefficient between ICGs and immune cell subsets. P-value is converted to -logI0. 
of stage_T, stage_N, stage_M, stage, sex and new event. The expression of all the 13 ICGs showed medium and low level (Supplementary Figure 2). Although the expression patterns of these genes were relatively consistent at different stages of cancer, we found that the numbers of stage_N, stage_M and Stage were significantly different between CD274, CD48 and so on (Figure 6, rank test $p<$ $0.05)$. However, no significance was observed between age, new event, and stage $\mathrm{T}$ and the 13 ICGs (Supplementary Figure 3).

\section{Subsets Defined by ICGs are Associated with Prognosis}

Based on the analysis of above, we found that CD48 in TCGA cohort was significantly associated with both prognosis and neoantigens of CRC. Moreover, CD48 was also significantly positively correlated with the CD8A, suggesting that CD48 may cause gene expression disorder of adaptive immune resistance pathways. Therefore, we investigated whether, or not, the expressions of CD48 and other three important biomarkers PD-L1 (CD274), CTLA4 and IDO1 gene combinations were associated with prognosis in CRC.

On account of the medium and low expression levels of IDO1, CD274, CTLA4 and CD48 in COAD samples, we distributed these genes into high expression $(\mathrm{H})$ and low (L) groups by gene expression level density. Then, we integrated $\mathrm{H} / \mathrm{L}$ expression groups of IDO1, CD274, CTLA4 and CD48, respectively. According to the median value of gene expression in COAD samples among patients, we divided these samples into four types to identify the prognostic correlation of ICGs genes. We then performed survival analysis of these three pairs of genotypic samples in TCGA cohort respectively. However, we did not observe any significant difference in OS among the four types of samples (Figure 7A-C). Then, by analyzing the best and worst prognostic samples in TCGA separately, we found that the prognosis of the group with low expression of CD48 and CD274 was significantly better than that of the group with high expression of CD48 and CD274; the prognosis of the group with low expression of CD48 and CTLA4 was marginally correlated with that of the group with high expression of CD48 and CTLA4: no significant prognostic difference was found between the group with high expression of CD48 and IDO1 and the group with low expression of CD48 and IDO1 (Figure 7D-F). Through further analysis, we found that it showed significant differences between the group with high expression of CD48 and IDO1 and the group with low expression of CD48 and IDO1 in the early stage of COAD (stage I and II) (Figure 7G-I).

\section{Enhancement of the Antitumor Activity of Anti-PD-LI in CRC by Si-B7-H4 is Dependent on NK Cells in vitro}

The above results show that VCTN1 (V-set domain-containing $\mathrm{T}$ cell activation inhibitor 1 ) was not only associated with poor outcomes in both databases, but also closely associated with TMB in CRC. To determine whether VCTN1 (B7-H4) is a potential therapeutic target for enhancing anti-PD-1/PD-L1 pathway activity, we first detected B7-H4 protein expression levels in various CRC cell lines. As shown in Figure 8A and B, HCT-116 and SW620 cells expressed high levels of B7-H4 both on the cell surface and in total protein levels. It is known that NK cells from the blood of cancer patients displayed higher expression of $\mathrm{B} 7-\mathrm{H} 4$ receptor than their counterparts in healthy donors. ${ }^{28} \mathrm{NK}$ cells are known to secrete IFN- $\gamma$ and other cytokines after activation by target cells. ${ }^{29,30}$ To examine the involvement of B7-H4 in modulating susceptibility to NK cell activity by anti-PD-L1, we transduced HCT-116 cells with B7-H4-siRNAs and RT-PCR and Western Blot were used to detect knockdown efficiency (Figure 8C). B7-H4-KD target cells were then tested for susceptibility to CD56+NK-92 cells combined with antiPD-L1. As functional markers of NK cell activity, we measured secretion of IFN- $\gamma$ (Figure 8D), the level of CD107a degranulation ${ }^{31}$ (Figure 8E) and NK-cell-induced apoptosis using AnnexinV/7AAD (Figure 8F). Compared with anti-PD-L1 alone, the anti-PD-L1 and reduced expression of B7-H4 in HCT-116 cells combination significantly increased IFN- $\gamma$ secretion by NK-92 cells ( $p$ $<0.05)$. In the presence of anti-PD-L1, NK-92 cells showed enhanced cytotoxicity on tumor cells following B7-H4-KD, as measured by CD107a degranulation and apoptosis. We then examined whether modulated B7-H4 expression on tumor target cells potentiated the anti-tumor activity of anti-PD-L1, we added anti-PD-L1 to co-cultures of NK cells with B7-H4-KD HCT-116 cells and measured tumor cell viability by MTT assay (Figure $8 \mathrm{G}$ ). Compared with anti-PD-L1 alone, the combination significantly reduced cell growth in HCT-116 cells. These 

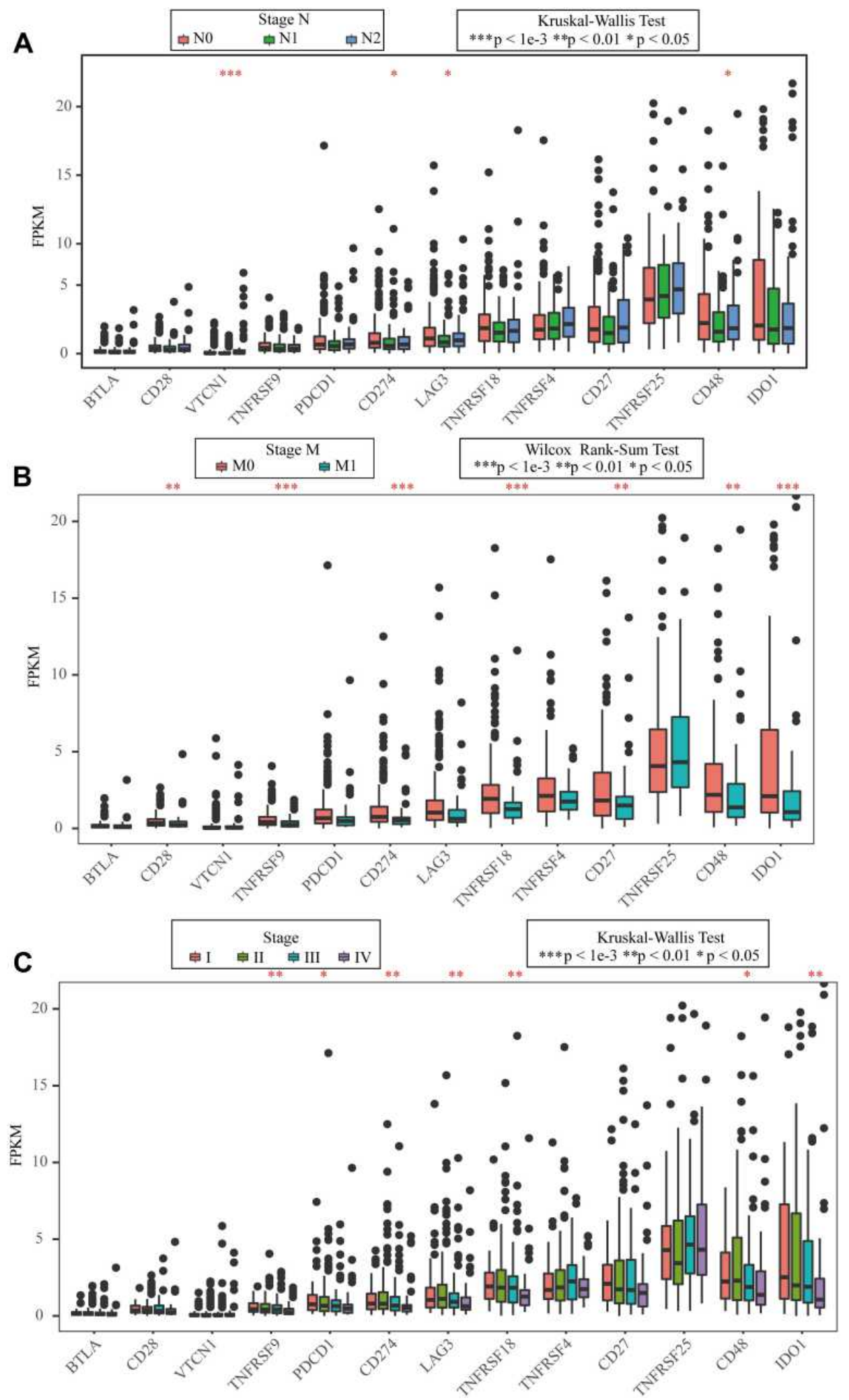

Figure 6 Association between ICGs and clinical features in CRC. (A) the FPKM boxplot of I I ICGs expressed on N stage; (B) the FPKM boxplot of I3 ICGs expressed on $M$ stage; (C) the FPKM boxplot of I3 ICGs expressed on stage (Data are plotted as mean \pm SD. $* P<0.05, * * P<0.01, * * * P<0.001$ ). 


\section{All stages}
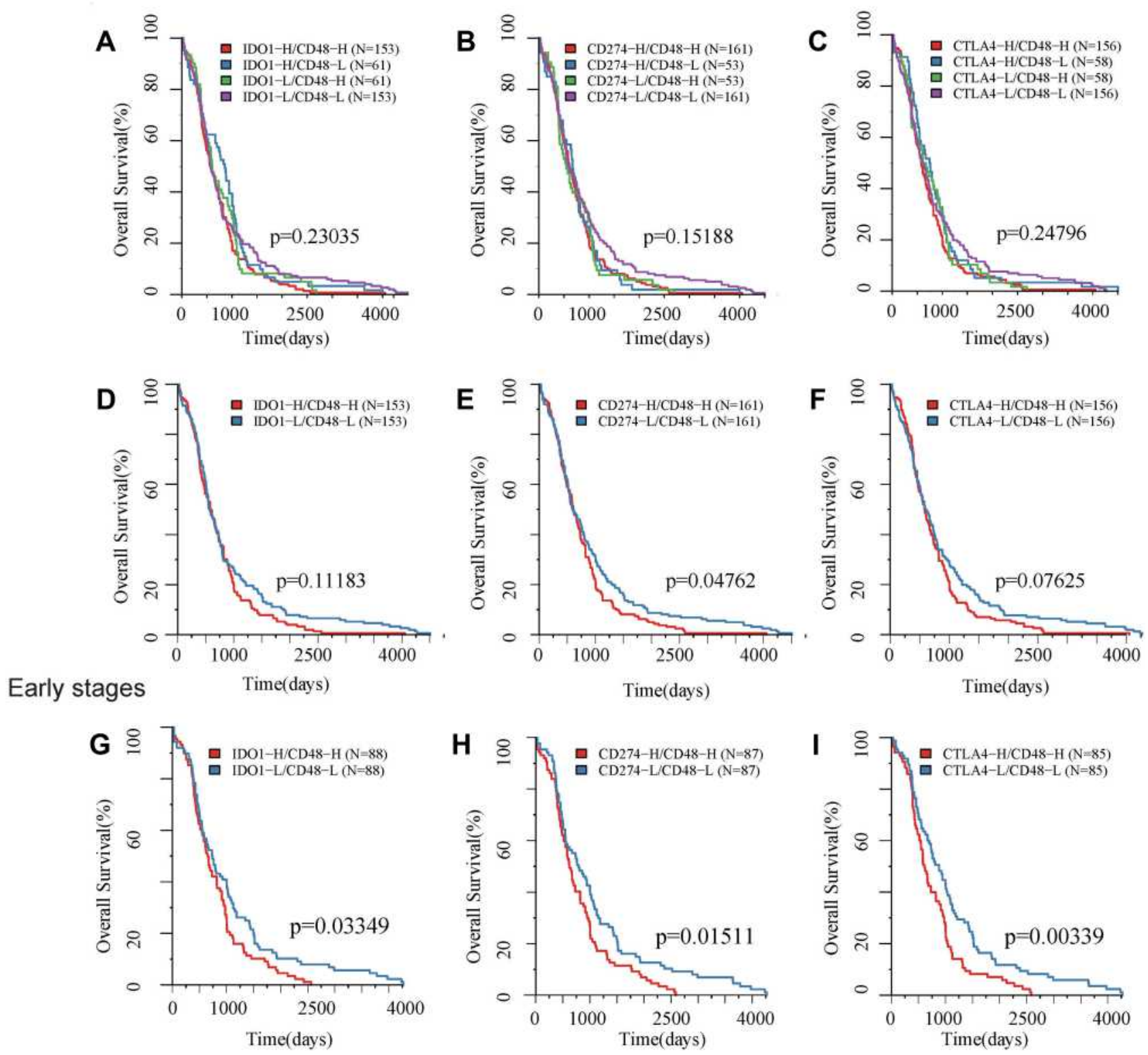

Figure 7 Kaplan- Meier survival curves for subtypes defined by ICGs associated with overall survival. Horizontal axis: overall survival time, days, Vertical axis: survival function. KM survival curve of TCGA based on (A) high/low expression of IDOI and CD48 grouping samples; (B) high/low expression of PD-LI (CD274) and CD48; (C) high/low expression of CTLA4 and CD48; (D) high expression of IDOI+CD48 and low expression of IDOI +CD48 in all patients. (E) high expression of PD-LI (CD274) + CD48 and low expression of PD-LI (CD274) +CD48 in all patients; (F) high expression of CTLA4 +CD48 and low expression of CTLA4+CD48 in all patients; (G) high expression of IDOI +CD48 and low expression of IDOI +CD48 in early stage patients; $(\mathbf{H})$ high expression of PD-LI (CD274) +CD48 and low expression of PD-LI $(C D 274)+C D 48$ in early stage patients; (I) high expression of CTLA4 +CD48 and low expression of CTLA4 +CD48 in early stage patients.

results indicate that $\mathrm{B} 7-\mathrm{H} 4-$ inhibition increases anti-PDL1-mediated NK cell cytotoxicity on CRC tumor cells.

\section{Discussion}

Immune checkpoint blockade (ICB) therapy has achieved remarkable clinical benefit in multiple cancers. Immune checkpoint molecule PD-L1 expression was correlated with survival benefits from anti-PD-1 therapy in most cancers, except for CRC. The vast majority of mCRC patients barely respond to current ICB monotherapy. ${ }^{32}$ In the present study, by using a computational approach, we identified the expression and combined evaluated of 47 immune checkpoint genes (ICGs) in CRC and these molecules can, in principal, become interesting targets for immunotherapy. We observed that several ICGs showed significant prognostic potential in CRC in both the TCGA 
A

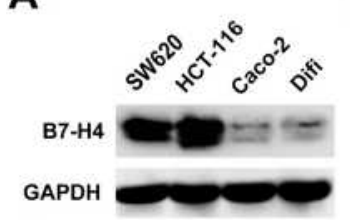

D

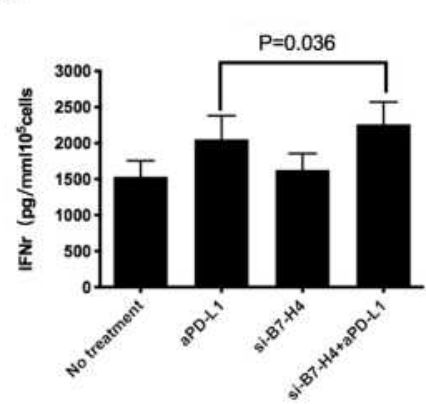

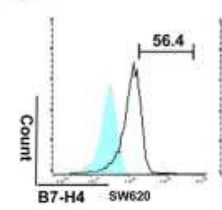

E

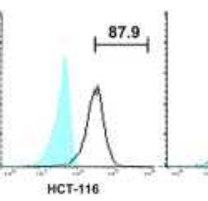

NK+HCT-116

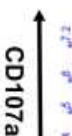

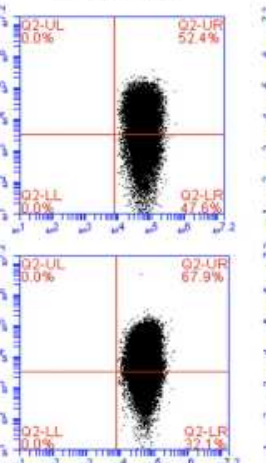

NK+HCT-116+aPD-L1 NK+siB7-H4-HCT-116 +aPD-L1

$\mathrm{NK}+\mathrm{siB} 7-\mathrm{H} 4-\mathrm{HCT}-116$

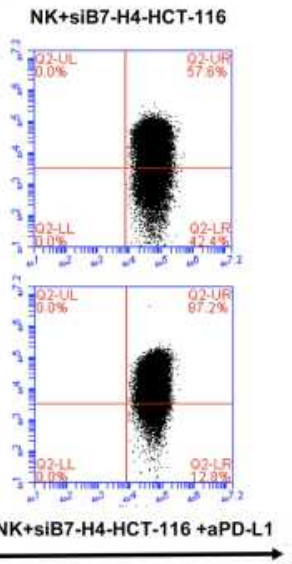

CD56

F
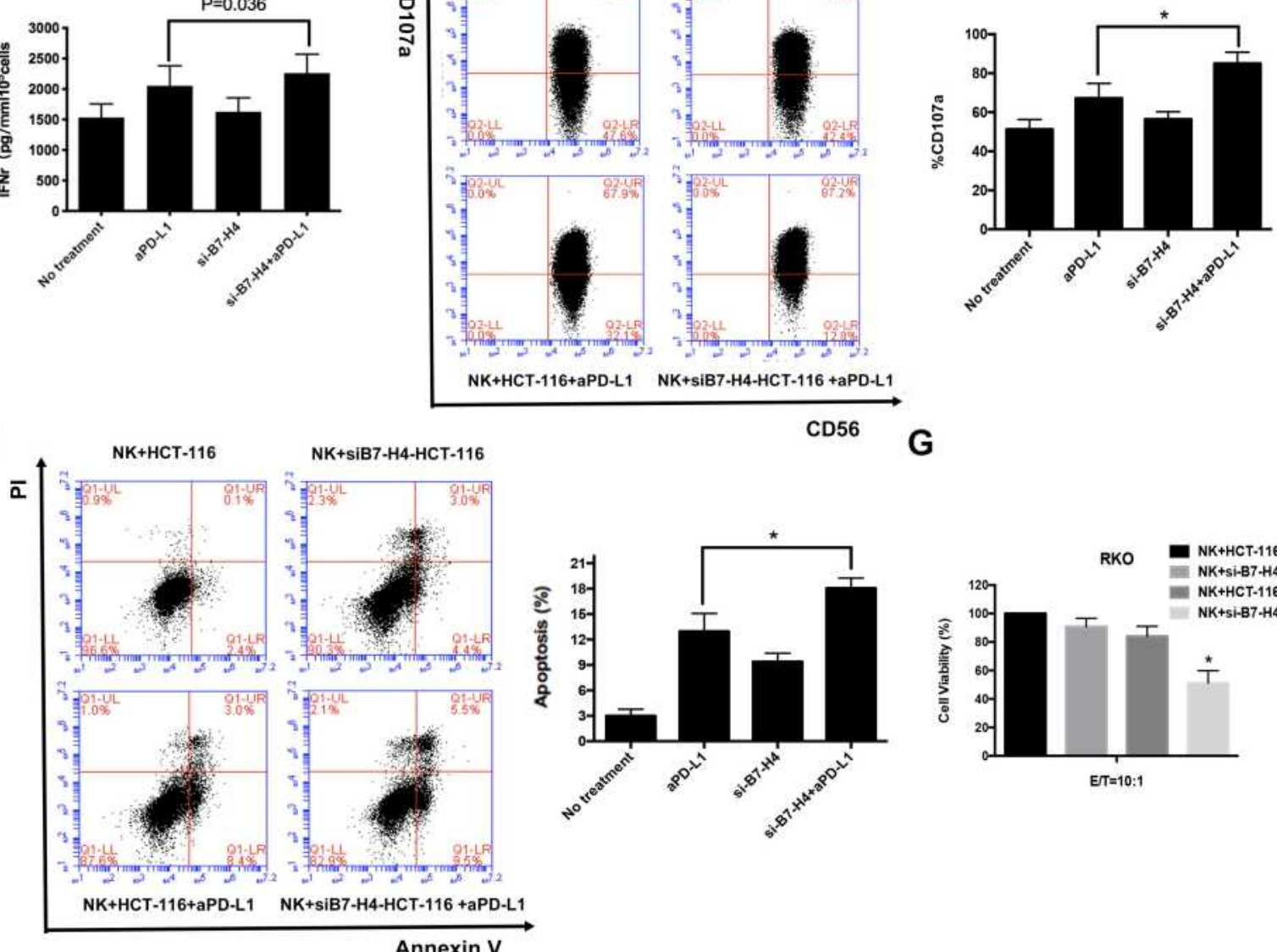

C

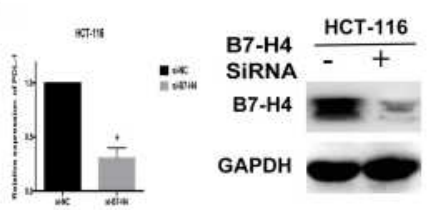

G

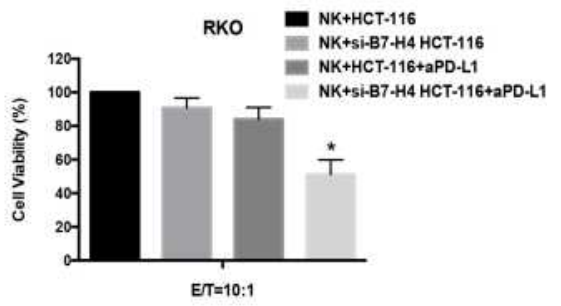

Figure 8 Reduced expression of B7-H4 enhances the antitumor activity of aPD-LI in CRC depending on NK cells in CRC cells in vitro. (A) B7-H4 total protein expression in 4 CRC cell lines. (B) The expression levels of B7-H4 on the surface of CRC cells are measured by flow cytometry. (C) HCT-II6 cells were transfected with either negative control (NC)-siRNA or B7-H4-siRNA for 48h. B7-H4 expression levels were detected by qRT-PCR and Western blot. Controls and B7-H4-KD cell lines were incubated with NK-92 cells pre-stimulated with 100 Units/mL IL-2 at I0:I E/T ratio as described in Methods. Incubated cells were treated with or without I0 $\mu$ g/mL PD-LI antibody SHR-1316 for 48 h. (D) Levels of IFN- $\gamma$ secretion in culture supernatant was detected by ELISA. (E) The percent NK cells expressing CDI07a and (F) the AnnexinV/7AAD positive B7-H4-KD and control cells mediated by NK-92 cells were measured by flow cytometry. (G) Viable of incubated cells were determined by MTT assay. Student's t-tests were used for statistical analyses. Data are plotted as mean \pm SD. *Indicated $p<0.05$.

and GEO databases. According to univariate Cox regression analysis, we identified that the VTCN1 gene was correlated with poor survival in CRC. VTCN1, can also be called B7-H4 which is a member of the B7 family of immune-regulatory proteins. This protein acts as a negative regulatory factor in the T cell-mediated immune response in the $\mathrm{TME}^{33}$ which has been detected in a variety of cancers and is associated with poor clinical prognosis. ${ }^{34}$ In $\mathrm{CRC}$, high expression of $\mathrm{B} 7-\mathrm{H} 4$ in the tumour stroma had higher survival rate than low B7-H4 patients, ${ }^{35}$ but because there was no significant correlation observed in cancer foci and the sample size was small, this study did not receive much attention. In the present study, we combined mRNAs expression of ICGs and clinical data from two databases to show that $\mathrm{B} 7-\mathrm{H} 4$ can predict survival in CRC. Moreover, anti-B7-H4 immunotherapy has demonstrated significant therapeutic efficacy in vivo $^{28}$ and to be synergistic with several existing therapies, ${ }^{36}$ showing its utility in combination treatment. Our experiments confirmed that down-regulation of B7-H4 increases anti-PD-L1-mediated NK cell cytotoxicity on CRC tumor cells. Now a series of early phase clinical trials are 
underway to explore the efficacy and safety of targeting B7-H4 in different cancers. ${ }^{37}$ So, it suggests that B7-H4 might be new CRC prognostic molecular biomarker and serve as a potential target for CRC immunotherapy.

Recent studies have revealed that checkpoint molecules may participate in function and signal, through relatively different pathways. ${ }^{38}$ In the CHECKMATE-142 trial, dual checkpoint blockade increased response rates in dMMR mCRC. ${ }^{15}$ At present, numerous additional combinations are being studied in PMMR and AMMR mCRC by clinical trials, however, little is known about the extent of cooperative interaction between checkpoints. Therefore, it is advantageous to investigate the interaction between checkpoints for better combination therapies. This study found that the expression levels of these 43 ICGs were strongly correlated with each other, which serves as the theoretical basis for combination checkpoint blockade.

We further investigated the associations between ICGs and other immunotherapy treatment biomarkers in CRC. The results indicated that the expression of a series of ICGs was significantly associated with both CRC prognosis and immunotherapy treatment biomarkers TMB, neoantigens and MMR. Previous studies show that positive PD-L1 expression in the tumour cells has been identified as a poor prognostic factor for survival in CRC. ${ }^{39,40}$ However, in the microenvironment, stromal PD-L1/PD-1 expression was associated with better OS and disease-free survival. $^{41}$ To date, dMMR, high TMB and neoantigen may be associated with better CRC specific survival. ${ }^{42-44}$ But it is still under investigation whether these immune markers are confirmed as a positive prognostic marker in mCRC. Interestingly, we found that a series of ICGs predicting poor prognosis, for example, B7-H4, IDO1, LAG3, CD48 and TNFRSF9 had a significant positive correlation with these immunotherapy treatment biomarkers. There is no conflict here. Immune markers are associated with better prognosis because these patients are better candidates for immunotherapy. Hence these patients have a good prognosis after treatment, rather than a good prognosis naturally. The positive correlation here is logical. The former refers to the up-regulation of PD-L1 expression as a result of the more aggressive biology of dMMR patients. ${ }^{45}$ Therefore, in combination with immunotherapy treatment biomarkers, ICG expression may be useful in prognostic stratification of CRC, and potentially identification of CRC cases that might respond to CPIs.

As a new component of a TNM-Immune classification of cancer, the Immunoscore consisting of three adaptive immune-resistance pathways markers (CD45RO, CD3 and CD8), is found to be a potential biomarker with which to assess the effectiveness of CPIs as is also associated with better survival in CRC. ${ }^{46-48}$ Although some famous ICG expressions have been interrogated in multiple cohorts of CRC, only a few studies have addressed the importance of ICGs expression in combination with CD8 and other adaptive immune-resistance pathway gene expressions that may contribute to ICG immunosuppression. ${ }^{43}$ Our results revealed that CD8A was significantly positively correlated with a series of ICGs indicating that the adaptive immune pathway gene has a certain regulatory effect on the expression of ICGs. A previous study showed that the expression of both PD-1 and PD-L1 in CRC ( $\mathrm{n}=87$ ) was inversely correlated with the tumour stage. ${ }^{49}$ In our study, a series of ICGs, including CD274 and CD48 had a significant correlation with lymph node stage, metastases stage and stage in CRC, which significantly decreased during the tumour stage. These results indicated that the expression of ICGs varied in different tumour stages and exhibited complex functions in tumour progression.

We then investigated the associations between ICGs and prognosis in CRC profoundly. Although PD1, PD-L1 and IDO1 were important immune-checkpoint genes and important biomarkers for immunotherapy, univariate survival analysis did not show that they alone were associated with significant prognosis in CRC. Signaling CD48 is an adhesion and costimulatory molecule expressed constitutively on most hematopoietic cells, particularly in antigen presenting cells (APC). CD48 binds to CD2 and is involved in a wide variety of innate and adaptive immune responses. ${ }^{50}$ The interaction between CD48 and CD244 has been reported to cause NK cell dysfunction in hepatocellular carcinoma, suggesting that CD48 may play an important role in mediating the immune response in both immune activation or suppression. ${ }^{51}$ Moreover, it has been reported that $\mathrm{CD} 48$ can suppress $\mathrm{CRC}$ progression by inhibiting the proliferation, migration, and invasion capacity of CRC cells as well as tumour growth in vivo. ${ }^{52} \mathrm{In}$ the present study, we mainly focused on the expression pattern of ICGs and clinical correlation in CRC, so we included these ICGs as the object. Kaplan-Meier's survival analysis confirmed that the low expression groups of IDO1, CD274, CTLA4 and CD48 had the best prognosis, while the high expression groups of IDO1, CD274, CTLA4 and CD48 had the worst prognosis. Based on current findings, ICGs subsets might play a role for the prognosis differences observed between risk groups as 
defined by our ICGs. We propose that ICGs subsets might complement risk biomarkers and improve the accuracy of prognostic prediction in CRC.

Furthermore, a novel immune-related gene signature has also been reported to provide some references for the clinical precision immunotherapy of patients. ${ }^{53}$ However, more and more studies have shown that immune checkpoints play a more important role in predicting prognosis and treatment of tumours. Therefore, our immune checkpoint-based analysis has greater clinical significance, especially for immunotherapy. There has also been a study on the relationship between immune checkpoint molecules with the tumor-infiltrating lymphocytes load, patient survival and a high mutation rate in CRC. ${ }^{54}$ Our research, however, takes a completely new perspective and analyze the association of ICGs with prognosis, immunotherapy biomarkers and signature genes related to immune activation in CRC. Additionally, we identified VTCN1and CD48 that are directly related to CRC survival and validated them by preclinical trials. Finally, here are some deficiencies of this study. Regrettably, we did not observe any significant differences in patients in stage III + stage IV divided by CTLA4 and IDO1, suggesting that these subtypes we found are more effective in predicting prognosis in early stage CRC. Meanwhile, whether CD48 can predict the prognosis of CRC still needs more experimental and clinical verification.

\section{Conclusion}

In summary, the gene expressions of 47 ICGs in CRC and their associations with clinical outcome and other immunotherapy treatment biomarkers were demonstrated. Both VTCN1 and CD48 showed a negative correlation with CRC specific survival and positive correlation with several immunotherapy biomarkers. Finally, we believe that VTCN1 and CD48 may serve as potential prognostic factors as well as therapeutic targets in CRC. Moreover, further illumination of the underlying mechanisms and the interactions among these ICGs may have important implications for the success of checkpoint blockade in CRC.

\section{Abbreviations}

ICGs, immune checkpoint genes; CRC, colorectal cancer; mCRC, metastatic CRC; OS, overall survival; TCGA, The Cancer Genome Atlas; TMB, tumor mutational burden; dMMR, different Mismatch Repair; MSI-H, microsatellite instability-high; PD-1, programmed death-1; PD-L1, programmed death ligand-1; CTLA-4, Cytotoxic T-Lymphocyte Antigen 4; pMMR, proficiency of MMR; MSS, microsatellite stability; PFS, progression-free survival; IFN $\gamma$, Interferon gamma; TMB, tumor mutational burden; IDO1, indoleamine-2,3-dioxygenase-1; CD274, cluster of differentiation 274; VTCN1, V-set domain-containing $\mathrm{T}$ cell activation inhibitor 1 ; CPIs, checkpoint inhibitors.

\section{Acknowledgments}

This research was supported by the National Key Research and Development Program of China (NO.2017YFC1308900); National Science and Technology Major Project of the Ministry of Science and Technology of China (No. 2017ZX09304025); National Natural Science Foundation of China (No.82003302); Technological Special Project of Liaoning Province of China (2019020176-JH1/103); Science and Technology Plan Project of Liaoning Province (NO.2013225585); The Key Research and Development Program of Liaoning Province (2018225060); Doctor Scientific Research Initiation Fund project of Liaoning Province (2020-BS-089). The authors would like to acknowledge the Key Laboratory of Precision Diagnosis and Treatment of Gastrointestinal Tumors, Ministry of Education (China Medical University, Shenyang, China) for providing the space and equipment for conducting the experiments.

\section{Disclosure}

The authors report no conflicts of interest in this work.

\section{References}

1. Ciardiello D, Vitiello PP, Cardone C, et al. Immunotherapy of colorectal cancer: challenges for therapeutic efficacy. Cancer Treat Rev. 2019;76:22-32. doi:10.1016/j.ctrv.2019.04.003

2. Ciombor KK, Wu C, Goldberg RM. Recent therapeutic advances in the treatment of colorectal cancer. Annu Rev Med. 2015;66(1):83-95. doi:10.1146/annurev-med-051513-102539

3. Angell H, Galon J. From the immune contexture to the immunoscore: the role of prognostic and predictive immune markers in cancer. Curr Opin Immunol. 2013;25(2):261-267. doi:10.1016/j.coi.2013.03.004

4. Gentles AJ, Newman AM, Liu CL, et al. The prognostic landscape of genes and infiltrating immune cells across human cancers. Nat Med. 2015;21(8):938-945. doi:10.1038/nm.3909

5. Xu F, Jin T, Zhu Y, et al. Immune checkpoint therapy in liver cancer. J Exp Clin Cancer Res. 2018;37(1):110. doi:10.1186/s13046-018$0777-4$

6. Marin-Acevedo JA, Dholaria B, Soyano AE, et al. Next generation of immune checkpoint therapy in cancer: new developments and challenges. J Hematol Oncol. 2018;11(1):39. doi:10.1186/s13045018-0582-8 
7. Liu SS, Yang YZ, Jiang C, et al. Comparison of immunological characteristics between paired mismatch repair-proficient and -deficient colorectal cancer patients. J Transl Med. 2018;16(1):195. doi:10.1186/s12967-018-1570-z

8. Raut CP, Pawlik TM, Rodriguez-Bigas MA. Clinicopathologic features in colorectal cancer patients with microsatellite instability. Mutat Res. 2004;568(2):275-282. doi:10.1016/j.mrfmmm.2004.05.025

9. Hampel H, Frankel WL, Martin E, et al. Screening for the lynch syndrome (hereditary nonpolyposis colorectal cancer). N Engl J Med. 2005;352(18):1851-1860. doi:10.1056/NEJMoa043146

10. Koopman M, Kortman GA, Mekenkamp L, et al. Deficient mismatch repair system in patients with sporadic advanced colorectal cancer. $\mathrm{Br}$ J Cancer. 2009;100(2):266. doi:10.1038/sj.bjc.6604867

11. Vilar E, Tabernero J. Molecular dissection of microsatellite instable colorectal cancer. Cancer Discov. 2013;3(5):502-511. doi:10.1158/ 2159-8290.CD-12-0471

12. Le DT, Uram JN, Wang H, et al. PD-1 blockade in tumors with mismatch-repair deficiency. $N$ Engl J Med. 2015;372 (26):2509-2520. doi:10.1056/NEJMoa1500596

13. U.S. Food \& Drug Administration. OPDIVO (nivolumab) injection, for intravenous use. Package Insert. 2019. Accessed October 16, 2019.

14. Overman MJ, Lonardi S, Wong KYM, et al. Durable clinical benefit with nivolumab plus ipilimumab in DNA mismatch repair-deficient/ microsatellite instability-high metastatic colorectal cancer. J Clin Oncol. 2018;36(8):773-779. doi:10.1200/JCO.2017.76.9901

15. Overman MJ, McDermott R, Leach JL, et al. Nivolumab in patients with metastatic DNA mismatch repair-deficient or microsatellite instability-high colorectal cancer (CheckMate 142): an open-label, multicentre, Phase 2 study. Lancet Oncol. 2017;18(9):1182-1191. doi:10.1016/S1470-2045(17)30422-9

16. Rittmeyer A, Barlesi F, Waterkamp D, et al. Atezolizumab versus docetaxel in patients with previously treated non-small-cell lung cancer (OAK): a Phase 3, open-label, multicentre randomised controlled trial. Lancet. 2017;389(10066):255-265. doi:10.1016/S01406736(16)32517-X

17. Daud AI, Loo K, Pauli ML, et al. Tumor immune profiling predicts response to anti-PD-1 therapy in human melanoma. J Clin Invest. 2016;126(9):3447-3452. doi:10.1172/JCI87324

18. Inomata M, Kado T, Okazawa S, et al. Peripheral PD1-positive CD4 T-lymphocyte count can predict progression-free survival in patients with non-small cell lung cancer receiving immune checkpoint inhibitor. Anticancer Res. 2019;39(12):6887-6893. doi:10.21873/ anticanres. 13908

19. Berntsson J, Eberhard J, Nodin B, et al. Expression of programmed cell death protein 1 (PD-1) and its ligand PD-L1 in colorectal cancer: relationship with sidedness and prognosis. Oncoimmunology. 2018;7 (8):e1465165. doi:10.1080/2162402X.2018.1465165

20. Wang J, Sanmamed MF, Datar I, et al. Fibrinogen-like protein 1 is a major immune inhibitory ligand of LAG3. Cell. 2019;176(12):334-347.e12. doi:10.1016/j.cell.2018.11.010

21. ElTanbouly MA, Croteau W, Noelle RJ, et al. VISTA: a novel immunotherapy target for normalizing innate and adaptive immunity. Semin Immunol. 2019;42:101308. doi:10.1016/j.smim.2019.101308

22. Das M, Zhu C, Kuchroo VK. Tim-3 and its role in regulating anti-tumor immunity. Immunol Rev. 2017;276(1):97-111. doi:10.1111/imr. 12520

23. Huang RY, Francois A, McGray AR, et al. Compensatory upregulation of PD-1, LAG-3, and CTLA-4 limits the efficacy of single-agent checkpoint blockade in metastatic ovarian cance. Oncoimmunology. 2017;6:e1249561. doi:10.1080/2162402X.2016.1249561

24. Danilova L, Ho WJ, Zhu Q, et al. Programmed Cell Death Ligand-1 (PD-L1) and CD8 expression profiling identify an immunologic subtype of pancreatic ductal adenocarcinomas with favorable survival. Cancer Immunol Res. 2019;7(6):886-895. doi:10.1158/2326-6066. CIR-18-0822
25. Xu Z, Dai J, Wang D, et al. Assessment of tumor mutation burden calculation from gene panel sequencing data. Onco Targets Ther. 2019;12:3401-3409. doi:10.2147/OTT.S196638

26. Ma R, Xu L, Qu X, et al. AZ304, a novel dual BRAF inhibitor, exerts anti-tumour effects in colorectal cancer independently of BRAF genetic status. Br J Cancer. 2018;118(11):1453-1463. doi:10.1038/ s41416-018-0086-x

27. Yarchoan M, Hopkins A, Jaffee EM. Tumor mutational burden and response rate to $\mathrm{PD}-1$ inhibition. $N$ Engl $J$ Med. 2017;377 (25):2500-2501. doi:10.1056/NEJMc1713444

28. Li J, Lee Y, Li Y, et al. Co-inhibitory molecule B7 superfamily member 1 expressed by tumor-infiltrating myeloid cells induces dysfunction of anti-tumor CD8+ $\mathrm{T}$ cells. Immunity. 2018;48 (4):773-786. doi:10.1016/j.immuni.2018.03.018

29. Farag SS, Caligiuri MA. Human natural killer cell development and biology. Blood Rev. 2016;20(3):123-137. doi:10.1016/j.blre.2005. 10.001

30. Vivier E, Tomasello E, Baratin M, et al. Functions of natural killer cells. Nat Immunol. 2008;9(5):503-510. doi:10.1038/ni1582

31. Alter G, Malenfant JM, Altfeld M. CD107a as a functional marker for the identification of natural killer cell activity. J Immunol Methods. 2004;294(1-2):15-22. doi:10.1016/j.jim.2004.08.008

32. Lee JJ, Chu E. Recent advances in the clinical development of immune checkpoint blockade therapy for Mismatch Repair Proficient (pMMR)/non-MSI-H metastatic colorectal cancer. Clin Colorectal Cancer. 2018;17(4):258-273. doi:10.1016/j.clcc.2018. 06.004

33. Podojil JR, Miller SD. Potential targeting of B7-H4 for the treatment of cancer. Immunol Rev. 2017;276(1):40-51. doi:10.1111/imr.12530

34. MacGregor HL, Ohashi PS. Molecular pathways: evaluating the potential for B7-H4 as an immunoregulatory target. Clin Cancer Res. 2017;23(12):2934-2941. doi:10.1158/1078-0432.CCR-15-2440

35. Qiu ZC, Wu JY, Wang Y, et al. Expression and clinical significance of negative costimulatory molecules B7-H1, B7-H3 and B7-H4 in the process of colorectal cancer's evolution. Transl Cancer Res. 2018;7 (4):1026-1035. doi:10.21037/tcr.2018.07.15

36. Leung J, St-Onge P, Stagg J, et al. Synergistic effects of host B7-H4 deficiency and gemcitabine treatment on tumor regression and anti-tumor $\mathrm{T}$ cell immunity in a mouse model. Cancer Immunol Immunother. 2017;66(4):491-502. doi:10.1007/s00262-016-1950-2

37. John P, Wei Y, Liu W, et al. The B7x immune checkpoint pathway: from discovery to clinical trial. Trends Pharmacol Sci. 2019;40 (11):883-896. doi:10.1016/j.tips.2019.09.008

38. Tu L, Guan R, Yang H, et al. Assessment of the expression of the immune checkpoint molecules PD-1, CTLA4, TIM-3 and LAG-3 across different cancers in relation to treatment response, tumor-infiltrating immune cells and survival. Int $J$ Cancer. 2020;147(2):12. doi:10.1002/ijc.32785

39. Yaghoubi N, Soltani A, Ghazvini K, et al. PD-1/ PD-L1 blockade as a novel treatment for colorectal cancer. Biomed Pharmacother. 2019;110:312-318. doi:10.1016/j.biopha.2018.11.105

40. Shen Z, Gu L, Mao D, et al. Clinicopathological and prognostic significance of PD-L1 expression in colorectal cancer: a systematic review and meta-analysis. World J Surg Oncol. 2019;17(1):4. doi:10.1186/s12957-018-1544-x

41. Wyss J, Dislich B, Koelzer VH, et al. Stromal PD-1/PD-L1 expression predicts outcome in colon cancer patients. Clin Colorectal Cancer. 2019;18(1):e20-e38. doi:10.1016/j.clcc.2018.09.007

42. Phipps AI, Limburg PJ, Baron JA, et al. Association between molecular subtypes of colorectal cancer and patient survival. Gastroenterology. 2015;148(1):77-87. doi:10.1053/j.gastro.2014.09. 038

43. Emambux S, Tachon G, Junca A, et al. Results and challenges of immune checkpoint inhibitors in colorectal cancer. Expert Opin Biol Ther. 2018;18(5):561-573. doi:10.1080/14712598.2018.1445222 
44. Giannakis M, Mu XJ, Shukla SA, et al. Genomic correlates of immune-cell infiltrates in colorectal carcinoma. Cell Rep. 2016;17 (4):1206. doi:10.1016/j.celrep.2016.10.009

45. Rosenbaum MW, Bledsoe JR, Morales-Oyarvide V, et al. PD-L1 expression in colorectal cancer is associated with microsatellite instability, BRAF mutation, medullary morphology and cytotoxic tumor-infiltrating lymphocytes. Mod Pathol. 2016;29(9):1104-1112. doi:10.1038/modpathol.2016.95

46. Mlecnik B, Tosolini M, Kirilovsky A, et al. Histopathologic-based prognostic factors of colorectal cancers are associated with the state of the local immune reaction. J Clin Oncol. 2011;29(6):610-618. doi:10.1200/JCO.2010.30.5425

47. Galon J, Pagès F, Marincola FM, et al. The immune score as a new possible approach for the classification of cancer. J Transl Med. 2012;10(1):1. doi:10.1186/1479-5876-10-1

48. Pagès F, Mlecnik B, Marliot F, et al. International validation of the consensus immunoscore for the classification of colon cancer: a prognostic and accuracy study. Lancet. 2018;391(10135):2128-2139. doi:10.1016/S0140-6736(18)30789-X

49. Gatalica Z, Snyder C, Maney T, et al. Programmed cell death 1 (PD-1) and its ligand (PD-L1) in common cancers and their correlation with molecular cancer type. Cancer Epidemiol Biomarkers Prev. 2014;23(12):2965-2970. doi:10.1158/1055-9965.EPI-14-0654
50. McArdel SL, Terhorst C, Sharpe AH. Roles of CD48 in regulating immunity and tolerance. Clin Immunol. 2016;164:10-20. doi:10.10 16/j.clim.2016.01.008

51. Wu Y, Kuang DM, Pan WD, et al. Monocyte/macrophage-elicited natural killer cell dysfunction in hepatocellular carcinoma is mediated by CD48/2B4 interactions. Hepatology. 2013;57(3):1107-1116. doi:10.1002/hep. 26192

52. Yuan YH, Chen J, Wang J, et al. Identification hub genes in colorectal cancer by integrating weighted gene co-expression network analysis and clinical validation in vivo and vitro. Front Oncol. 2020;10:638. doi: $10.3389 /$ fonc. 2020.00638

53. Zhang X, Zhao H, Shi X, et al. Identification and validation of an immune-related gene signature predictive of overall survival in colon cancer. Aging. 2020;12(24):26095-26120. doi:10.18632/ aging. 202317

54. Kitsou M, Ayiomamitis GD, Zaravinos A. High expression of immune checkpoints is associated with the TIL load, mutation rate and patient survival in colorectal cancer. Int $J$ Oncol. 2020;57 (1):237-248. doi:10.3892/ijo.2020.5062

\section{Publish your work in this journal}

OncoTargets and Therapy is an international, peer-reviewed, open access journal focusing on the pathological basis of all cancers, potential targets for therapy and treatment protocols employed to improve the management of cancer patients. The journal also focuses on the impact of management programs and new therapeutic agents and protocols on patient perspectives such as quality of life, adherence and satisfaction. The manuscript management system is completely online and includes a very quick and fair peer-review system, which is all easy to use. Visit http://www.dovepress.com/ testimonials.php to read real quotes from published authors. 